\title{
The fast molecular outflow in the Seyfert galaxy IC 5063 as seen by ALMA
}

\author{
Raffaella Morganti ${ }^{1,2}$, Tom Oosterloo ${ }^{1,2}$, J. B. Raymond Oonk ${ }^{1,3}$, Wilfred Frieswijk ${ }^{1}$, and Clive Tadhunter ${ }^{4}$ \\ 1 ASTRON, the Netherlands Institute for Radio Astronomy, Postbus 2, 7990 AA, Dwingeloo, The Netherlands \\ e-mail: morganti@astron.nl \\ 2 Kapteyn Astronomical Institute, University of Groningen, PO Box 800, 9700 AV Groningen, The Netherlands \\ ${ }^{3}$ Leiden Observatory, Leiden University, PO Box 9513, 2300 RA Leiden, The Netherlands \\ ${ }^{4}$ Department of Physics and Astronomy, University of Sheffield, Sheffield, S7 3RH, UK
}

Received 10 February 2015 / Accepted 22 May 2015

\begin{abstract}
We use high-resolution $(0.5 \operatorname{arcsec}) \mathrm{CO}(2-1)$ observations performed with the Atacama Large Millimetre/submillimetre Array to trace the kinematics of the molecular gas in the Seyfert 2 galaxy IC 5063. The data reveal that the kinematics of the gas is very complex. A fast outflow of molecular gas extends along the entire radio jet $(\sim 1 \mathrm{kpc})$, with the highest outflow velocities about $0.5 \mathrm{kpc}$ from the nucleus, at the location of the brighter hot spot in the western lobe. The ALMA data show that a massive, fast outflow with velocities up to $650 \mathrm{~km} \mathrm{~s}^{-1}$ of cold molecular gas is present, in addition to the outflow detected earlier in warm $\mathrm{H}_{2}$, $\mathrm{HI}$ and ionized gas. All phases of the gas outflow show similar kinematics. IC 5063 appears to be one of the best examples of the multi-phase nature of AGN-driven outflows. Both the central AGN and the radio jet could energetically drive the outflow, however, the characteristics of the outflowing gas point to the radio jet being the main driver. This is an important result because IC 5063, although one of the most powerful Seyfert galaxies, is a relatively weak radio source $\left(P_{1.4 \mathrm{GHz}}=3 \times 10^{23} \mathrm{~W} \mathrm{~Hz}^{-1}\right)$. All the observed characteristics can be described by a scenario of a radio plasma jet expanding into a clumpy medium, interacting directly with the clouds and inflating a cocoon that drives a lateral outflow into the interstellar medium. This model is consistent with results obtained by recent simulations. A stronger, direct interaction between the jet and a gas cloud is present at the location of the brighter western lobe. This interaction may also be responsible for the asymmetry in the radio brightness of the two lobes. Even assuming the most conservative values for the conversion factor $\mathrm{CO}-\mathrm{to}-\mathrm{H}_{2}$, we find that the mass of the outflowing gas is between 1.9 and $4.8 \times 10^{7} M_{\odot}$, of which between 0.5 and $1.3 \times 10^{7} M_{\odot}$ is associated with the fast outflow at the location of the western lobe. These amounts are much larger than those of the outflow of warm gas (molecular and ionized) and somewhat larger than of the H I outflow. This suggests that most of the observed cold molecular outflow is due to fast cooling after being shocked. This gas is the end product of the cooling process, although some of it could be the result of only partly dissociated clouds. Our $\mathrm{CO}$ observations demonstrate that fast outflows of substantial masses of molecular gas can be driven by relativistic jets, although in the case of IC 5063 the outflows are not fast enough to remove significant amounts of gas from the galaxy and the effects are limited to the central $\sim 0.5 \mathrm{kpc}$ from the centre.
\end{abstract}

Key words. galaxies: active - galaxies: individual: IC 5063 - ISM: jets and outflows - radio lines: galaxies

\section{Introduction}

The discovery of the large variety of structures and phenomena traced by cold gas in early-type galaxies has made these objects much more complex than historically perceived. Although not a complete surprise (see e.g. the work of van Driel \& van Woerden 1991; and Wiklind et al. 1995), the plethora of new results, made possible by the technical improvements in the available and new observing facilities, has opened new and important avenues for the study of formation and evolution of early-type galaxies using this component of their interstellar medium (ISM; see e.g. Young et al. 2011; Serra et al. 2012, 2014; Davis et al. 2013; Alatalo et al. 2013).

In addition to being an effective tracer of the formation history of galaxies, cold gas has revealed unexpected characteristics that have allowed us to use it also for tracing extreme energetic phenomena like massive and fast AGN-driven gas outflows (Morganti et al. 2005a,b, 2013a,b; Feruglio et al. 2010; Dasyra \& Combes 2012; Mahony et al. 2013; Morganti 2015, and references therein). This finding has challenged our ideas of how the energy released by an AGN may interact with its surroundings (see e.g. Tadhunter 2008; Fabian 2013; Combes et al. 2015, for reviews on the topic). Cold gas has been found to represent the dominant mass component of the outflows observed (see e.g. Morganti et al. 2005a,b, 2013a,b; Kanekar \& Chengalur 2008; Holt et al. 2009; Feruglio et al. 2010; Alatalo et al. 2011; Dasyra \& Combes 2011, 2012; Cicone et al. 2014; García-Burillo et al. 2014, for some examples). This makes them particularly interesting for their potential impact on the ISM of the host galaxy. In the effort to understand the mechanisms that can regulate black hole growth and quenching star formation in early-type galaxies, the discovery, in a growing number of objects, of these AGN-driven massive outflows represents an important step towards building a more complete and realistic view of how feedback may work. Thanks to detailed studies of these phenomena in nearby objects, better constraints (i.e. distribution and kinematics of the outflowing gas, energy injected into the ISM, etc.) can be provided to theoretical models of galaxy formation that now routinely include feedback effects.

Different mechanisms have been proposed to accelerate the gas. Although (broad) winds from the accretion disk, launched by radiation pressure, interacting and shocking the surrounding 
medium is often favoured (e.g. Zubovas \& King 2012, 2014; Costa et al. 2014; Cicone et al. 2014), the role of the radio plasma has also gained interest. Indeed, this mechanism can be particularly relevant in early-type galaxies where up to $30 \%$ of the high-mass galaxies are radio loud (Best et al. 2005). A number of studies (Birzan et al. 2008; Cavagnolo et al. 2010; McNamara $\&$ Nulsen 2012) have shown a high efficiency of the coupling between radio plasma and the surrounding ISM and inter-galactic medium (IGM) and mechanical power of the radio jets exceeding the synchrotron power. These results suggest that this mechanism can also be relevant for relatively weak radio sources (see e.g. NGC 1266 and NGC 1433, Alatalo et al. 2011; Combes et al. 2013, respectively).

Expanding the number of known outflows of atomic (HI) and molecular gas to provide better statistics is the focus of a number of recent studies (see e.g. Gereb et al. 2014, 2015; Cicone et al. 2014). However, the other important direction where progress needs to be made is to spatially resolve the outflows and image the distribution, kinematics, and the physical properties of the gas in detail. In the case of $4 \mathrm{C} 12.50$, VLBI observations have, for the first time, allowed this to be established for the H I component (Morganti et al. 2013a). For the warm molecular gas, the most spectacular and convincing example of a jet-driven outflow has been found in IC 5063 by Tadhunter et al. (2014). In this object, the warm component of the molecular gas has been identified at the location of the interaction between the radio jet and the ISM, $0.5 \mathrm{kpc}$ from the central AGN, where an H I outflow was also found (Oosterloo et al. 2000). The AGN-driven outflows of cold molecular gas have so far only barely been resolved (e.g. Feruglio et al. 2010; Cicone et al. 2014) not always allowing a detailed modelling of the outflowing gas and its conditions. The possible exceptions to this are M 51 (Matsushita et al. 2007, 2015) and NGC 4258 (Martin et al. 1989; Krause et al. 2007). The situation is now changing with the advent of ALMA. The detailed case of NGC 1068 (García-Burillo et al. 2014) is an example of the dramatic improvement afforded by ALMA.

All together, these studies are the first to provide details on the physical parameters and the conditions of the gas in the outflows. One of these objects, the Seyfert 2 galaxy IC 5063, is the target of the ALMA observations presented here showing, for the first time, the complexity of the kinematics of the cold gas under the effect of radio jets.

\section{A case study: IC 5063}

IC 5063 is a Seyfert 2 object hosted by an early-type galaxy with a prominent dust lane, which is seen edge on. Together with NGC 1068, it is one of the most radio bright Seyfert objects with a power ${ }^{1}$ of $P_{1.4 \mathrm{GHz}}=3 \times 10^{23} \mathrm{~W} \mathrm{~Hz}^{-1}$ (Tadhunter et al. 2014). In the radio continuum, IC 5063 shows a triple structure of about 4 arcsec in size (about $0.93 \mathrm{kpc}$; see Morganti et al. $1998)$ aligned with the dust lane. H I emission $\left(8.4 \times 10^{9} M_{\odot}\right)$ is traced in a regularly rotating warped disk with radius $\sim 2$ arcmin (about $28 \mathrm{kpc}$ ), which is associated with the system of dust lanes. The likely counterpart of the H I disk is also detected in $\mathrm{CO}$ as originally observed with SEST in $\mathrm{CO}(1-0)$ by Wiklind et al. (1995) and, more recently, with APEX in CO(2-1) by Morganti et al. (2013b).

\footnotetext{
1 Assuming a Hubble constant $H_{0}=70 \mathrm{~km} \mathrm{~s}^{-1} \mathrm{Mpc}^{-1}$ and $\Omega_{\Lambda}=0.7$ and $\Omega_{\mathrm{M}}=0.3$, we use an angular scale distance to IC 5063 of $47.9 \mathrm{Mpc}$, implying a scale of $1 \operatorname{arcsec}=232 \mathrm{pc}$.
}

IC 5063 was the first object in which a fast, AGN-driven massive outflow of H I was detected (Morganti et al. 1998). This outflow was found to be located against the brightest radio hot spot, about $0.5 \mathrm{kpc}$ from the radio core (Oosterloo et al. 2000), which has been interpreted as evidence that the outflow is driven by the interaction of the radio jet with the surrounding ISM in which the jet is moving in the plane of the large-scale gas disk of IC 5063. This hypothesis has been further strengthened by observations (using ISAAC on the VLT) of warm molecular gas $\left(\mathrm{H}_{2}\right.$ at $\left.2.2 \mu \mathrm{m}\right)$ which have, for the first time, shown the presence of outflowing molecular gas co-spatial with the bright radio hot spot (Tadhunter et al. 2014). A signature of outflow of cold molecular gas has been found using $\mathrm{CO}(2-1)$ observations with APEX (Morganti et al. 2013b). The limited spatial resolution of these observations did not allow us to determine where the outflow is occurring in relation to the radio structure and the main goal of the ALMA data presented in this paper is to remedy this situation. The data presented here complement the information about ionized (Morganti et al. 2007), warm molecular (Tadhunter et al. 2014), and cold atomic (Morganti et al. 1998; Oosterloo et al. 2000), making IC 5063 the best case of a multi-phase AGN-driven outflow studied so far.

The sensitivity and spatial resolution of the new ALMA observations allow us to show for the first time the detailed distribution and kinematics of the cold molecular fast outflow, providing a major step forward in the understanding of the interaction between the energy released by the AGN and the surrounding medium.

\section{The ALMA data}

IC 5063 was observed during Cycle 1 on the 27 May 2014 with the Atacama Large Millimeter/submillimeter Array (ALMA) using Band 6, covering simultaneously the $\mathrm{CO}(2-1)$ centred on $227.968 \mathrm{GHz}$ and two additional base-bands (at 232 and $247 \mathrm{GHz}$ ) for imaging of the continuum. The observations were pointed at the nucleus of IC 5063, with a single-pointing field of view (FoV) of 27", making use of the correlator in Frequency Division Mode with a $1.875 \mathrm{GHz}$ bandwidth (corresponding to $2466 \mathrm{~km} \mathrm{~s}^{-1}$ at the frequency of $\left.\mathrm{CO}(2-1)\right)$. The frequency resolution was $976.5 \mathrm{kHz}\left(1.28 \mathrm{~km} \mathrm{~s}^{-1}\right)$, but channels were combined when making the image cube to provide a better match to the observed line widths. The observation made use of 31 antennas with a maximum baseline of $1.5 \mathrm{~km}$. The duration of the observations was $1 \mathrm{~h}$, which was required to reach the sensitivity to locate and trace the outflow of molecular gas. The initial calibration was done in $\mathrm{CASA}^{2}$ (v4.2 McMulline et al. 2007) using the ALMA reduction scripts. These calibrated $u v$ data were subsequently exported to MIRIAD ${ }^{3}$ (Sault et al. 1995), which was used to perform additional self-calibration and significantly improved the quality of the images. All further reduction steps (continuum subtraction, mapping/cleaning) were also done in MIRIAD.

The data cubes were made using various Briggs weightings to explore the optimum for imaging, however, the images did not show any major differences (owing to the good $u v$ coverage of ALMA). Here we present the final data cubes obtained using Briggs robust $=0$. The line cube was produced using a velocity resolution, after Hanning smoothing, of $20 \mathrm{~km} \mathrm{~s}^{-1}$. At this velocity resolution, the rms noise per channel is $\sim 0.3 \mathrm{mJy} \mathrm{beam}^{-1}$. The large array configuration used provides a resolution of the

\footnotetext{
2 http://casa.nrao.edu/

3 http://www .atnf.csiro.au/computing/software/miriad/
} 


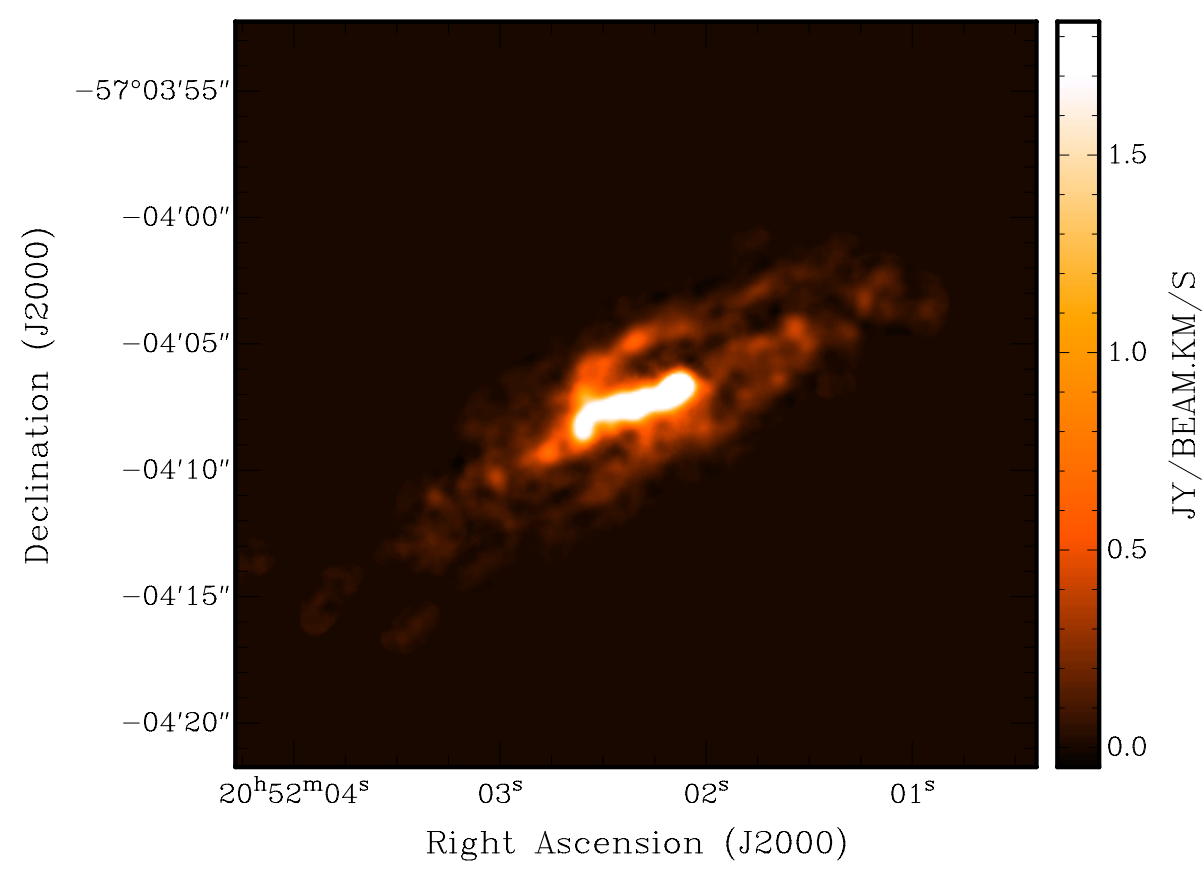

Fig. 1. Total intensity image representing the distribution of the $\mathrm{CO}(2-1)$ in IC 5063 and showing the striking brightness contrast between the inner, bright $\mathrm{CO}$ and the fainter outer disk. The details of the bright inner region can be seen in Fig. 2. The resolution of this image is $0.54 \times 0.45 \operatorname{arcsec}\left(\mathrm{PA}=-31.7^{\circ}\right)$. This image is not corrected for the primary beam of ALMA. line data of $0.54 \times 0.45 \operatorname{arcsec}\left(\mathrm{PA}=-31.7^{\circ}\right)$, nicely allowing the line emission to be resolved in relation to the radio continuum emission.

In Fig. 1 the total intensity of the $\mathrm{CO}(2-1)$ is shown. The single pointing ALMA observation detects molecular gas up to a radius of $\sim 10^{\prime \prime}$, therefore covering only the very inner part $(r<2 \mathrm{kpc})$ of the Hi disk observed with ATCA (Morganti et al. 1998). The bulk of the emission covers the same range of velocities (between 3140 to $3630 \mathrm{~km} \mathrm{~s}^{-1}$ ) as traced by the APEX observations (Morganti et al. 2013b). However, the wider band of ALMA allows us to trace a much broader component of blueshifted gas, going down to velocities of $\sim 2750 \mathrm{~km} \mathrm{~s}^{-1}$, i.e. up to $\sim 650 \mathrm{~km} \mathrm{~s}^{-1}$ blueshifted compared to the systemic velocity.

A continuum image was also produced from two additional basebands using uniform weighting. The rms noise of the continuum image is $30 \mu \mathrm{Jy}$ beam $^{-1}$. The restoring beam $0.45 \times 0.42$ arc$\sec \left(\mathrm{PA}=-30 \cdot 4^{\circ}\right)$. Figure 2 shows the comparison between the $\mathrm{mm}$ continuum and the molecular gas in the inner region.

Finally, the CS(5-4) line at $242.205 \mathrm{GHz}$ was included in a fourth base-band, but no emission from this spectral line was detected. The noise level in this cube, not quite uniform across the band, ranges between 0.3 and $0.4 \mathrm{mJy}^{\text {beam }^{-1}}$.

\section{Results}

The main result of our observations is the detailed view of the complexity of the distribution and kinematics of the gas in the central regions. The data clearly demonstrate that, apart from the regularly rotating large-scale disk, the distribution and kinematics of the gas in the inner regions is strongly affected by the radio jet.

Figure 1 shows that the distribution of the $\mathrm{CO}(2-1)$ follows two structures: a high surface brightness inner structure of about the size of the radio continuum and a lower surface brightness, larger disk. This large disk is likely the molecular counterpart of the H I disk known to be present in this galaxy (see Sect. 4.3). Some of the gas in this disk appears to be distributed along arm-like features, however, the relatively edge-on orientation of

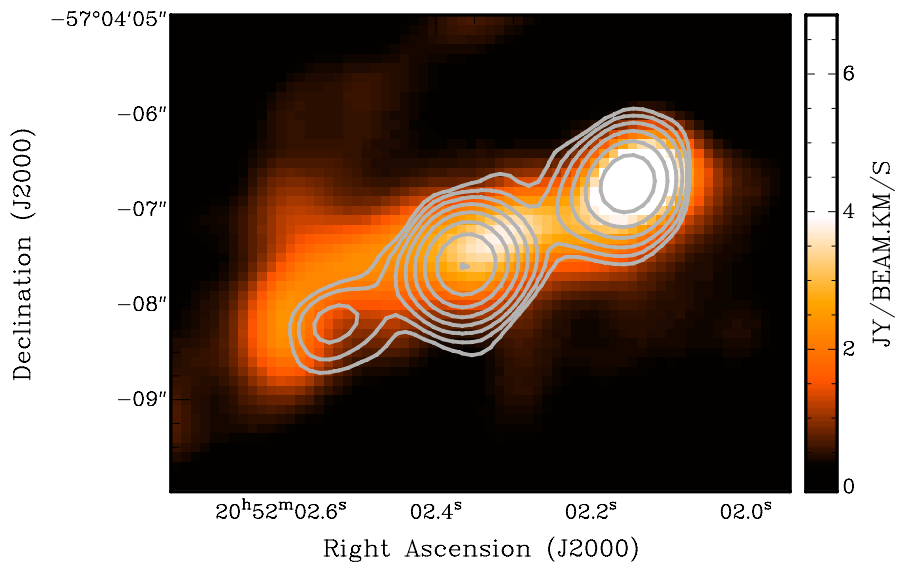

Fig. 2. Contours of the $230 \mathrm{GHz}$ continuum emission super-posed onto the central region of the total intensity of the $\mathrm{CO}(2-1)$ illustrating the spatial correlation between the two. Contour levels are 90, 180, 360, $720,1440,2880,5760$, and $15120 \mu \mathrm{Jy} \mathrm{beam}^{-1}$.

IC 5063 makes it difficult to identify and characterise these structures well.

The bright and elongated structure observed in the central regions shows a striking correspondence with the radio continuum, providing the first clear signature of the impact of the radio plasma on the ISM. Figure 2 illustrates how the $\mathrm{CO}$ bright emission wraps around the radio continuum, with the brightest $\mathrm{CO}$ component coincident with the brighter radio lobe on the western side.

Below we describe kinematics and other properties of these CO components.

\subsection{A complex, extended outflow of molecular gas}

The kinematics of the gas provides a striking view of what is happening in the central region. In Fig. 3 we show a positionvelocity $(p v)$ diagram of the $\mathrm{CO}$ gas taken along the major axis of the galaxy, which coincides, to within a few degrees, with the 


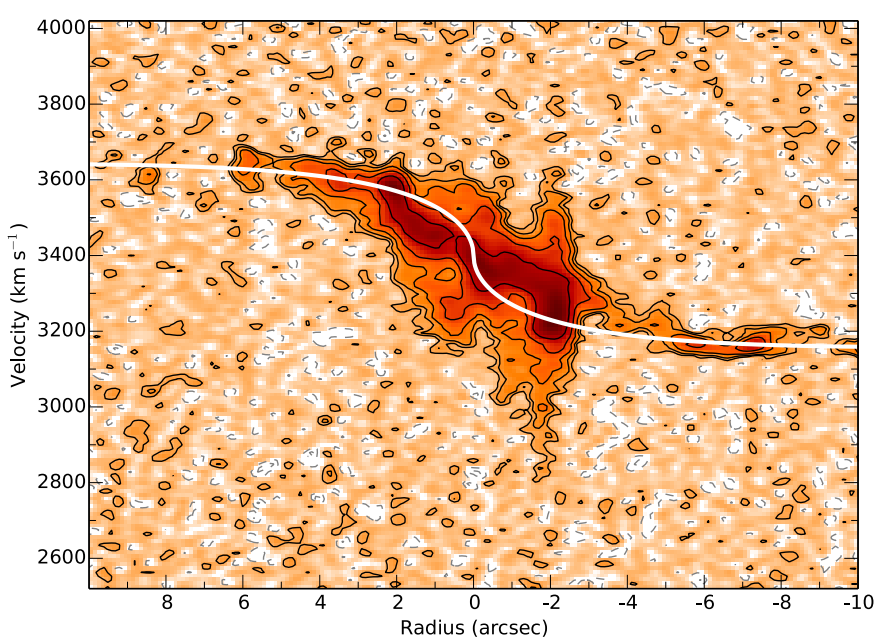

Fig. 3. Integrated position-velocity map taken along the major axis of IC 5063 in which the data were integrated over a length of 2 arcsec in the direction perpendicular to the major axis. The white line gives the rotation curve we derived from the photometry of Kulkarni et al. (1998), and indicates the expected kinematics of gas following regular rotation. Contour levels are $-3,-1.5,1.5,3,6,12$, and $24 \sigma$. Positive radii are east of the centre.

orientation of the radio continuum source. Given the relatively edge-on orientation of the system, a position-velocity diagram gives a better view of the kinematics of the gas compared to a velocity field. To make this $p v$ diagram, we have integrated the data over a length of $2^{\prime \prime}$ in the direction perpendicular to the major axis. Therefore this map shows all the gas in the vicinity of the radio source, but some of the gas associated with the largescale disk is not included. To indicate what can be expected for the kinematics of regularly rotating gas, we have over-plotted a rotation curve based on the HST photometry of IC 5063 of Kulkarni et al. (1998, see below).

Figure 3 clearly illustrates the strikingly complex kinematics of the gas in the region co-spatial with the radio plasma. Beyond a radius of $2^{\prime \prime}$ (i.e. $\sim 460 \mathrm{pc}$ ), the gas follows the expected rotation pattern fairly well and this gas corresponds to the large-scale disk, unaffected by the radio plasma. If gas with complex kinematics would have been present outside the region of the radio emission, the sensitivity of our observations would be able to see it. Instead, the change between complex to regular kinematics is quite abrupt. However, inside this radius, most of the gas has velocities very different from what is expected based on the rotation curve. In fact, a significant fraction of the gas is found in the so-called "forbidden quadrants" of the $p v$ diagram, i.e. those parts where co-rotating gas cannot appear. Examples of this are the gas at $r \sim-2^{\prime \prime}$ and with velocities around $3500 \mathrm{~km} \mathrm{~s}^{-1}$ and, similarly, the gas at $r \sim 1^{\prime \prime}$ and $V \sim 3200-3300 \mathrm{~km} \mathrm{~s}^{-1}$. In addition, for $-2^{\prime \prime}<r<0^{\prime \prime}$, there is a substantial amount of gas with velocities that are well outside the range of expected velocities, indicating that the kinematics of this gas is not determined by gravity alone. This gas is most likely related to the fast, outflowing H I gas that was discovered in absorption by Morganti et al. (1998). As we show below (Sect. 5.2), the distribution and kinematics of the molecular gas with anomalous velocities unequivocally point to the presence of outflowing gas and can be understood in terms of a lateral outflow driven by the radio jet.

In further agreement with earlier observations, also of other gas phases (Morganti et al. 2007, 1998; Tadhunter et al. 2014; Oosterloo et al. 2000), the most extreme velocities are confirmed

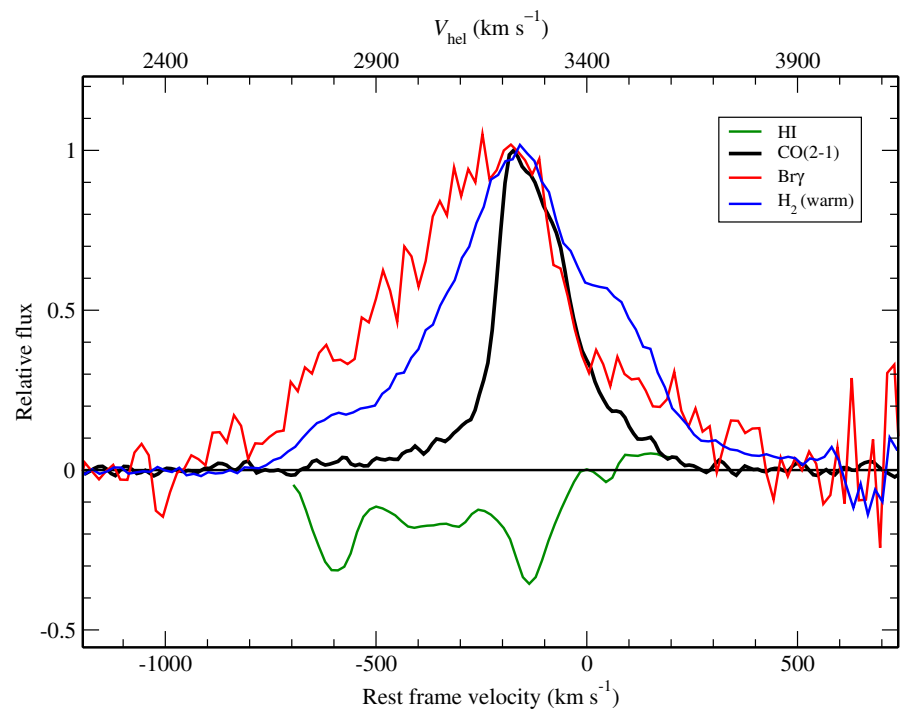

Fig. 4. Normalised spectra of $\mathrm{H}_{2}$ 1-0 $\mathrm{S}(1)$ (black solid line) and Brackett-gamma (Tadhunter et al. 2014) and CO (this work) integrated over the western radio lobe of IC 5053 are compared with the spatially integrated HI 21-cm absorption (from Morganti et al. 1998). The plot shows that all the components have gas reaching velocities of $-650 \mathrm{~km} \mathrm{~s}^{-1}$ or beyond.

to be present at the location of the bright $\mathrm{W}$ radio lobe, about $0.5 \mathrm{kpc}$ from the nucleus. This, again, is a strong indication that the most likely origin of these extreme kinematics must be the jet. The most extreme velocities of the anomalous gas are blueshifted by more than $500 \mathrm{~km} \mathrm{~s}^{-1}$ and redshifted by more than $400 \mathrm{~km} \mathrm{~s}^{-1}$ w.r.t. the regularly rotating gas at that location. The velocity profiles of the different gas components integrated over the region of the western hot spot are compared in Fig. 4. This is the case except for the HI, which shows the profile integrated over the full radio continuum region, although the most blueshifted HI absorption is most likely to occur only against the continuum of the bright $\mathrm{W}$ hot spot. In all cases (H I absorption, the ionized gas and the warm and cold molecular gas), the blueshifted component of the gas reaches velocities of $\sim-700 \mathrm{~km} \mathrm{~s}^{-1}$ (Morganti et al. 1998, 2007; Tadhunter et al. 2014, respectively).

The rotation velocity of IC 5063 is about $240 \mathrm{~km} \mathrm{~s}^{-1}$, which means that the escape velocity in the inner regions is likely to be above $550 \mathrm{~km} \mathrm{~s}^{-1}$ (Binney \& Tremaine 2008). We observe basically no gas with such extreme velocities (see Fig. 3). This means that, allowing for projection effects, at most a very small fraction of the anomalous gas leaves IC 5063, and that any possible effect on future star formation has to be through an increased energy input in the ISM and not through gas removal. A similar situation has been observed to be the case in NGC 1266 (Alatalo et al. 2015).

\subsection{The bright inner $\mathrm{CO}(2-1)$ distribution}

A characteristic of the $\mathrm{CO}(2-1)$ distribution that clearly appears from Fig. 1, as well as from the position-velocity diagram in Fig. 3 is that, although the large-scale, regular rotating disk is clearly seen, the emission from the region around the radio jet is much brighter in $\mathrm{CO}(2-1)$. Two possibilities can be suggested to explain this.

The bright inner CO $(J=2-1)$ emission in IC 5063 can be excited by a variety of physical processes, such as shocks 
(e.g. Hollenbach \& McKee 1989), cosmic rays (e.g. Sternberg et al. 1997; Ferland et al. 2009; Meijerink et al. 2011; Bayet et al. 2011), mechanical heating (Loenen et al. 2008; Meijerink et al. 2011) and photodissociation (including X-rays; e.g. Tielens \& Hollenbach 1985; Maloney et al. 1996; Meijerink et al. 2007). Given that we measured only one molecular line (CO $J=2-1$ ) at sufficient spatial resolution to resolve the molecular gas in this region, we are not able to discriminate between these different processes.

The difference between the inner and outer regions may reflect differences in the temperature and/or excitation of the molecular gas in the two regions because of the influence of the jet/cloud interaction. As discussed by Bolatto et al. (2013) for starbursts and ULIRGs, to first order, higher gas temperatures yield brighter $\mathrm{CO}$ emission, decreasing $X_{\mathrm{CO}}$ for the regions affected by the interaction. However, the increase in density may partly compensate for this effect, as pointed out by Bolatto et al. (2013). Therefore a certain level of compensation may occur, lessening the impact of environment on the conversion factor. Without a detailed study of the physical conditions of the molecular gas, e.g. using the full CO ladder, it is not possible to fully quantify these effects.

On the other hand, the kinematics of the gas clearly indicates that the effect of the radio jet is relevant for the distribution of the gas in the inner regions. The cocoon of shocked material created by the jet moving through the ISM may be able to push the gas and move it outwards. This explanation would be consistent with what is proposed by theoretical models (e.g. Wagner \& Bicknell 2011) and we consider this scenario in more detail in Sect. 5.2.

This scenario also implies that shocks likely play a prominent role in the heating and excitation of the molecular gas. In addition, the outwards moving jet and associated shocks boost the ionizing flux of cosmic rays (CR). Several theoretical studies have shown that high CR rates can significantly modify the chemistry and conditions of the molecular gas (e.g. Ferland et al. 2009; Bayet et al. 2011; Meijerink et al. 2011).

\subsection{The regularly rotating $\mathrm{CO}$ disk}

Outside the region of the radio continuum emission, the $\mathrm{CO}(2-1)$ shows regular kinematics. The characteristics are of a rotating disk with the western side approaching. This structure most likely represents the molecular counterpart of the regular disk already observed in $\mathrm{HI}$ and in ionized gas (Colina et al. 1991; Morganti et al. 1998, 2007). Indeed, the rotation velocities at large radii of this molecular gas are very similar to those of the H I, with an amplitude of the rotation of $\sim 246 \mathrm{~km} \mathrm{~s}^{-1}$. However, given the limited field of view of the primary beam of ALMA, we cannot determine the full extent of the molecular component of the large-scale disk. Furthermore, the comparison with the velocity profiles of the $\mathrm{H} \mathrm{I}$ in the inner regions is limited by the low-spatial resolution of the available H I observations. Large disks and rings of regularly rotating molecular gas are now known to be relatively common structures in early-type galaxies and are observed in more than $50 \%$ of CO detected early-type galaxies (Alatalo et al. 2013). Thus, in this respect, IC 5063 is not an exception.

We modelled the rotation of the regular $\mathrm{CO}$ distribution using the HST photometry of Kulkarni et al. (1998). This photometry, in the bands $F 110 \mathrm{~W}, F 160 \mathrm{~W}$, and $F 222 M$, shows that the unobscured light distribution of the inner regions of IC 5063 can accurately be described using the de Vaucouleurs' law. Figure 3 shows, over-plotted on a $p v$ diagram, the rotation curve expected for the observed light distribution where we have assumed that the light traces the mass distribution and that the mass distribution is spherical. The horizontal scale of the rotation curve is set by the observed effective radius ( $21^{\prime \prime} .5$ corresponding to $4.3 \mathrm{kpc}$ ), while we set the amplitude of the rotation curve to match the rotation velocities of the very outer regions in Fig. 3 .

\subsection{Molecular gas masses}

An important property that can be derived from the ALMA data, are the masses of the various kinematical components of the molecular gas. The integrated total flux (after primary beam correction) corresponds to $76.4 \pm 3.8 \mathrm{Jy} \mathrm{km} \mathrm{s}^{-1}$. The flux of the gas in the region co-spatial with the radio is $38.9 \pm 1.9 \mathrm{Jy} \mathrm{km} \mathrm{s}^{-1}$, of which $10.0 \pm 0.5 \mathrm{Jy} \mathrm{km} \mathrm{s}^{-1}$ are associated with gas with anomalous velocities; i.e gas with observed velocities incompatible with those of a regularly rotating disk with the rotation curve shown in Fig. 3, i.e. gas rotating faster than expected and gas that shows apparent counter rotation. The anomalous gas at the location of the $\mathrm{W}$ lobe has a flux of about $2.7 \pm 0.3 \mathrm{Jy} \mathrm{km} \mathrm{s}^{-1}$.

To convert these fluxes to masses of molecular gas, a number of assumptions are needed. As for the APEX data presented in Morganti et al. (2013b), we estimate the $\mathrm{H}_{2}$ masses by assuming a luminosity ratio $L^{\prime} \mathrm{CO}(2-1) / L^{\prime} \mathrm{CO}(1-0)=1$, typical of thermalised and optically thick gas, which could be the situation in the denser and warmer gas associated with an outflow.

For the kinematically disturbed gas, we considered a range of values for the $X_{\mathrm{CO}}$ conversion factor. This factor is assumed to be between $0.4 \times 10^{20} \mathrm{~cm}^{-2}\left(\mathrm{~K} \mathrm{~km} \mathrm{~s}^{-1}\right)^{-1}\left(\alpha_{\mathrm{CO}}=0.8\right)$, typical of a thick and dense medium and commonly used for ULIRG, and $0.2 \times 10^{20} \mathrm{~cm}^{-2}\left(\mathrm{~K} \mathrm{~km} \mathrm{~s}^{-1}\right)^{-1}$ (corresponding to $\alpha_{\mathrm{CO}}=0.34$, the most conservative case) derived for optically thin cases and suggested for turbulent gas associated with winds (see the discussion in Bolatto et al. 2013 and in Geach et al. 2014). Using these two values for the $\mathrm{CO}-\mathrm{to}-\mathrm{H}_{2}$ conversion factor, we derive masses for the anomalous molecular gas, ranging between 1.9 and $4.8 \times 10^{7} M_{\odot}$, of which between 0.5 and $1.3 \times 10^{7} M_{\odot}$ is associated with the gas with the most extreme kinematics at the location of the $\mathrm{W}$ lobe. The result is, therefore, that we confirm that the molecular outflow is more massive than the H I outflow, which was estimated to be $3.6 \times 10^{6} M_{\odot}$ (Morganti et al. 2007). We also confirm the large difference between the mass of the outflow of cold molecular gas, estimated here, and the mass of the warm molecular gas estimated by Tadhunter et al. (2014).

From the total integrated flux of the $\mathrm{CO}(2-1)$, we derive the total mass of the molecular gas of the regularly rotating gas outside the region of the radio plasma to be in the range $M_{\mathrm{H}_{2}}=1.0-1.1 \times 10^{9} M_{\odot}$, assuming an $X_{\mathrm{CO}}$ conversion factor equal to $2 \times 10^{20} \mathrm{~cm}^{-2}\left(\mathrm{~K} \mathrm{~km} \mathrm{~s}^{-1}\right)^{-1}$ (corresponding to $\alpha_{\mathrm{CO}}=4.6$, typical for the Milky Way). We consider the masses derived from the ALMA data to be more reliable measurements than those obtained from the APEX data (Morganti et al. 2013b). Two reasons should be considered. First of all, the blueshifted wing of $\mathrm{CO}$ was covering most of the available band in the APEX data. This made the definition of the continuum and the consequent continuum subtraction very uncertain (as discussed already in Morganti et al. 2013b). The large observing band of the ALMA data has solved this problem. Furthermore, the distribution and kinematics of the anomalous gas as observed in the ALMA data has turned out much more complex than was assumed when measuring the gas outflow from APEX by Morganti et al. (2013b). 
Table 1. Flux densities and spectral indices for the radio three radio continuum components in IC 5063.

\begin{tabular}{lccc}
\hline \hline Region & $\begin{array}{c}17 \mathrm{GHz} \\
\mathrm{mJy}\end{array}$ & $\begin{array}{c}228 \mathrm{GHz} \\
\mathrm{mJy}\end{array}$ & $\alpha$ \\
\hline $\mathrm{E}$ & 5.6 & 0.58 & 0.87 \\
Core & 30.8 & 12.8 & 0.33 \\
$\mathrm{~W}$ & 91.2 & 6.2 & 0.93 \\
\hline
\end{tabular}

\subsection{The radio continuum}

The continuum image at $230 \mathrm{GHz}$ is shown in contours in Fig. 2 overlaid to the zoom-in of the central region of the $\mathrm{CO}(2-1)$. The morphology of the continuum obtained from the ALMA data, a triple structure with the core in the middle and two lobes of which the western lobe is the brightest, is very similar to the structure observed at lower frequencies ( 17 and $24 \mathrm{GHz}$ with the ATCA) presented and discussed in Morganti et al. (2007). Using the present and the previous radio continuum observations, we derived the spectral indices of the three components. The total fluxes of the three components at 17 and $228 \mathrm{GHz}$ are listed in Table 1 together with the derived spectral indices. Between $17 \mathrm{GHz}$ and $228 \mathrm{GHz}$ we find a fairly steep spectral index for the two lobes ( $\alpha=0.87$ for the E lobe and $\alpha=0.93$ for the W lobe; $\left.S \propto v^{-\alpha}\right)$ and a flatter spectral index for the core $(\alpha=0.33)$. Thus the values obtained are similar, although slightly steeper, than found between the much closer frequencies of the ATCA data. As expected, these values support the non-thermal origin of the emission with the power law extending to the high frequencies of ALMA. The spectral indices also confirm the core and lobe classification of the three structures.

As mentioned above, the coincidence between the radio continuum and the distribution of the bright molecular gas is clear. The $\mathrm{CO}(2-1)$ gas wraps around the $\mathrm{E}$ lobe and part of the W lobe, while the brighter $\mathrm{CO}$ part appears to overlap (in projection) with the $\mathrm{W}$ hot spot. The bright $\mathrm{CO}$ region shows a strong correspondence with the radio continuum in the part dominated by the most kinematically disturbed gas. As discussed in Sect. 4.2, this can influence both the CO excitation as well as production of CR.

The radial distribution of the brightness of $\mathrm{CO}$ and radio continuum shows an offset in the location of the peaks. The peaks of $\mathrm{CO}$ are at slightly larger distances from the centre compared to the peaks of the radio continuum on both sides of the radio source; see Fig. 5. We comment on this further below; see Sect. 5.1.

\section{Discussion}

The observed distribution and kinematics of the cold molecular gas suggest that the radio plasma jet is driving the bulk of the gas outflow. Therefore, we present a simple model, which aims to explain these characteristics following the scenario presented in the numerical simulations of Wagner \& Bicknell (2011) and Wagner et al. (2012). These simulations describe the effects of a newly formed radio jet when moving though a dense clumpy medium. A porous medium with dense clumps forces the jet to find the path of least resistance, while interacting and gradually dispersing the dense clouds away from the jet axis. In this way, clouds can be accelerated to high velocities and over a wide range of directions and, along the path of the jet, a turbulent cocoon of expanding gas forms farther away from the jet axis.

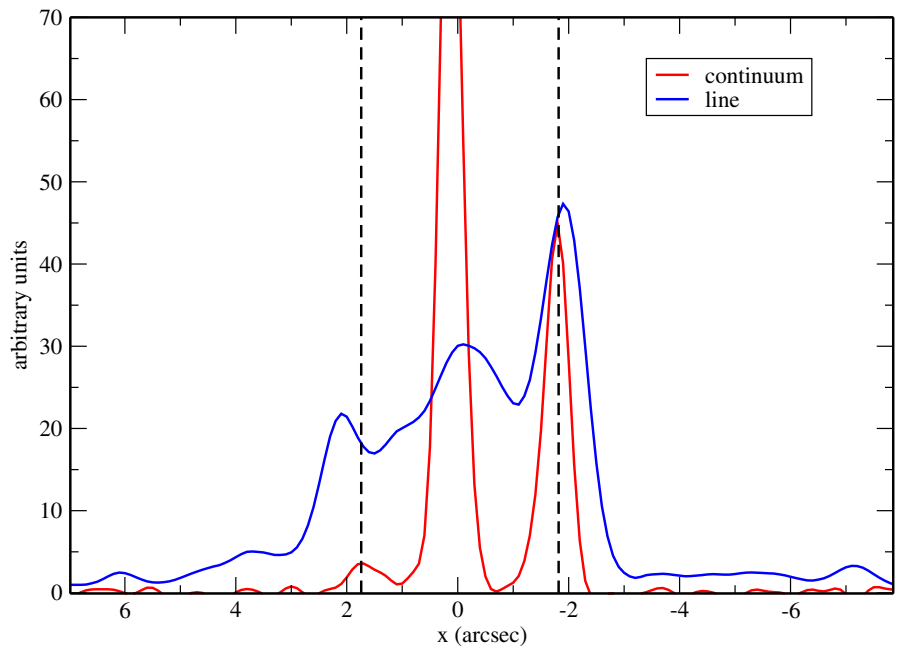

Fig. 5. Brightness distribution, in arbitrary units, along the direction of the radio jet of the synchrotron continuum emission (red line) and of the $\mathrm{CO}(2-1)$ emission (blue line). The dashed vertical lines indicate the peaks of the continuum hot spots, showing that the bright CO slightly extends beyond the hot spots.

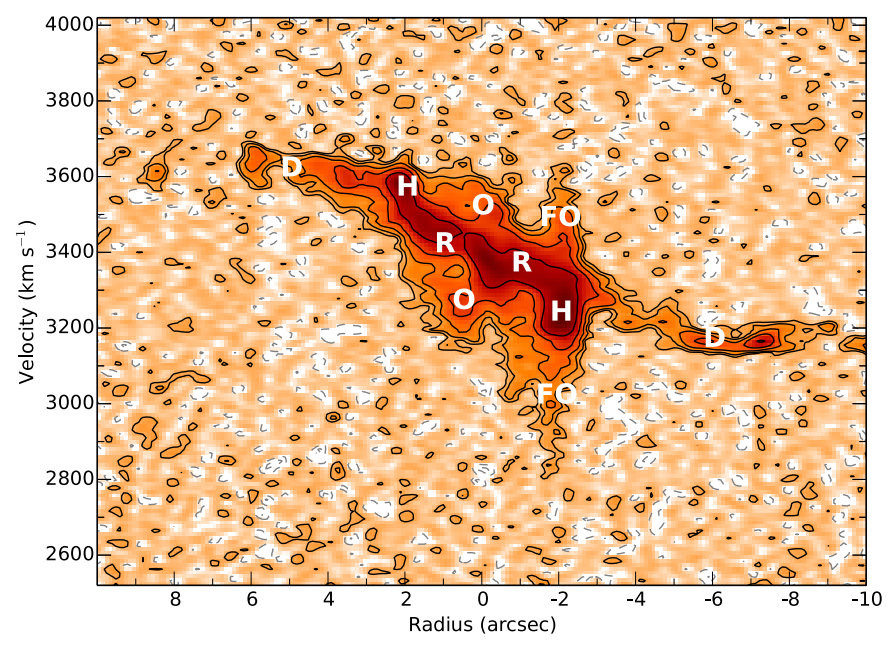

Fig. 6. Position-velocity diagram, as in Fig. 3, with the labels of various components identified (described in text).

Before describing the model, first we attempt to find clues to properties of the molecular gas in the observed kinematics as given in Fig. 3.

\subsection{Signatures of jet-ISM interaction in the kinematics of the molecular gas in the inner region of IC 5063}

A closer look at Fig. 3 shows that a number of key features can be identified in the molecular gas. These are illustrated in Fig. 6. Beyond radii of $2^{\prime \prime}$ (i.e. beyond the radio source), we detect the large-scale gas disk (labelled $D$ ), undisturbed by the radio jet. At smaller radii, a bright $\mathrm{S}$-shaped feature is seen. We suggest that this bright feature actually consists of two structures. One of these is a linear feature, of about 4 arcsec in size, which we labelled $R$ in Fig. 6. When linear features are observed in $p v$ diagrams of edge-on galaxies, such as IC 5063, they usually correspond to a rotating, ring-like structure in the galaxy, or to a disk with solid-body rotation. Solid-body rotation is not expected in the central regions of a massive early-type galaxy such as IC 5063 (e.g. Cappellari et al. 2013, see also Fig. 3), so it is 
more likely that $R$ corresponds to a ring-like structure. However, Fig. 3 shows that if $R$ corresponds to a ring, its rotation velocity has to be lower than the rotation speed expected from the mass model. We suggest that this may indicate that feature $R$ corresponds to a rotating ring-like structure with a radius of about $2^{\prime \prime}$, but rotating at velocities below nominal rotation because of the interaction with the outflowing gas.

At both ends of $R$, there is bright emission (labelled $H$ ) near the location of the two radio lobes, but the difference with $R$ is that they seem to rotate at the expected rotation speed. Together with feature $R$, these give the $\mathrm{S}$-shape of the bright emission inside $r=2^{\prime \prime}$. This may indicate that just outside the ring-like structure $R$ near the hot spots, there is bright emission, but it is rotating at the nominal rotation speed. This is also seen in Fig. 5, which shows that the bright molecular gas at the locations of $H$ extends slightly beyond the radio hot spots. The excitation conditions of the gas just beyond the hot spots, as seen from the centre, may already be changed by radiation from the jetISM interaction. However, the kinematics has not been affected yet and the gas is still rotating near the nominal rotation velocity. The overall kinematics shows that component $H$ only occurs near the radio lobes.

The other feature we identify is the fainter gas inside $r=2^{\prime \prime}$ with velocities (very) inconsistent with the nominal rotation (component $O$ ). In particular, part of this component has "forbidden" velocities, which implies that the kinematics of this component is not dominated by rotation and that it must involve a fast radial component. At the location of the $\mathrm{W}$ hot spot, the largest deviations from rotation are seen (component $F O$ ).

\subsection{Modelling of the kinematics of the gas}

Based on the discussion given above, we attempt to make a geometrical model of the gas kinematics in IC 5063. The basic idea of the model is that a plasma jet is expanding in the plane of the gas disk of IC 5063 and interacting with this gas disk. This interaction is pushing the gas away from the centre and creating a cocoon of laterally expanding gas along the radio jet.

To model the data cube, we employ a geometrical modelling technique often used for modelling the kinematics of gas disks in galaxies (e.g. Józsa et al. 2007), i.e. we describe the gas disk in IC 5063 by a set of concentric elliptical rings where each ring has an orientation, a density, and a rotation- and radial velocity. We stress that we attempt to only geometrically describe the kinematics of the gas; the model does not include any physics to describe, e.g. the excitation of the gas or the actual interaction of the jet plasma with the gas disk. For the inclination of the rings, we choose $80^{\circ}$ and for the position angle $115^{\circ}$.

The face-on layout of the model is shown in Fig. 7 and illustrates how IC 5063 would be seen from one of its poles. Along the radio jet, the cocoon around the jet drives a lateral expansion (indicated by the black vertical arrows) of the gas of the inner gas disk (light brown ellipse in Fig. 7) away from the jet axis. The interaction between the expanding cocoon and the inner gas disk also causes the rotation velocity in this region to be well below the nominal rotation, as we seem to observe in Fig. 3. This gas would correspond to component $O$. At the radius where this laterally expanding gas meets the undisturbed outer disk, a ring-like interface is formed of material with lower rotation velocities, but the expansion is halted. We identify this with component $R$. The gas of the quiescent, large-scale disk outside the zone of influence (dark brown Fig. 7) is rotating according to the full rotation expected from the mass distribution (Fig. 3). The horizontal white arrow Fig. 7 indicates the jet direction. The

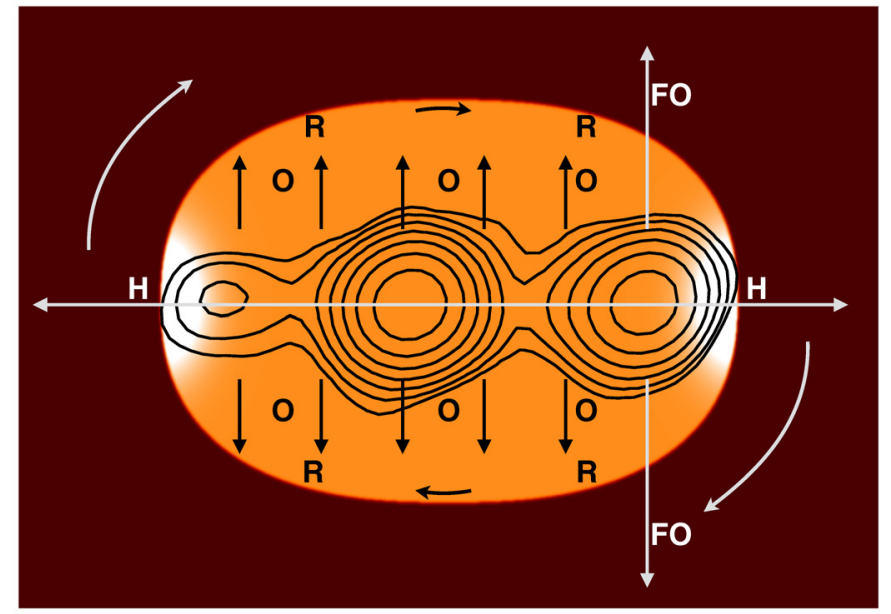

Fig. 7. Schematic illustration of the face-on view of the model described in Sect. 5.2, with the contours of the continuum emission.

vertical white arrows represent the extreme outflow velocities at the location of the $\mathrm{W}$ hot spot because of the direct interaction of the jet with the ISM (component $F O$ ). Just beyond the two hot spots, the rotation of the gas in the ISM follows our mass model, but has higher brightness because of the nearby hot spot (component $H$ ). We also include this component to reproduce the observation that the molecular appears to extend somewhat beyond the bright radio continuum source (see Fig. 5).

We attempted to reproduce the observed $p v$ diagram of IC 5063 by constructing data cubes produced by models of the kind described above. Figure 3 shows that the kinematics and distribution of the gas is not fully symmetric so any symmetric model has difficulty fitting the data in detail. Therefore we did not attempt to replicate the fine details of the brightness distribution and consequently the quality of a fit is not assessed using a numerical goodness-of-fit criterion such as, e.g. a $\chi^{2}$. Instead, we have focussed on whether a model data cube reproduces the locations in the $p v$ diagram where gas is detected and where it is not.

We also attempted to keep the number of free parameters required to make an acceptable match with the data in the sense described in the previous paragraph to a minimum. For example, since we access the validity of a model by a qualitative comparison with the data, the density/emissivity of the gas of components $O$ and $R$ does not have to be a free parameter and is assumed to be uniform (i.e. not to depend on position). In this way, the model provides a better test for the assumed kinematics and geometry.

The main two parameters we found to be important to describe the data, are the velocity of the lateral expansion of component $O$ and the reduced rotation velocity of component $R$. Initially, we also varied the orientation of the jet, w.r.t. the line of sight. We find, however, this parameter to be strongly degenerate with the outflow velocity of component $O$ in order to reproduce the observed (projected) velocities of component $O$; i.e. for a jet oriented more in the direction of the line of sight, we have to increase the lateral expansion velocity to obtain the same projected velocity, while not changing the qualitative aspects of the model $p v$ diagrams. We have therefore chosen to consider only models for which the jet is moving in the plane of the sky; these models give a lower limit to the expansion velocity of $O$. For simplicity, we do not include the large-scale disk $D$ in our models. 


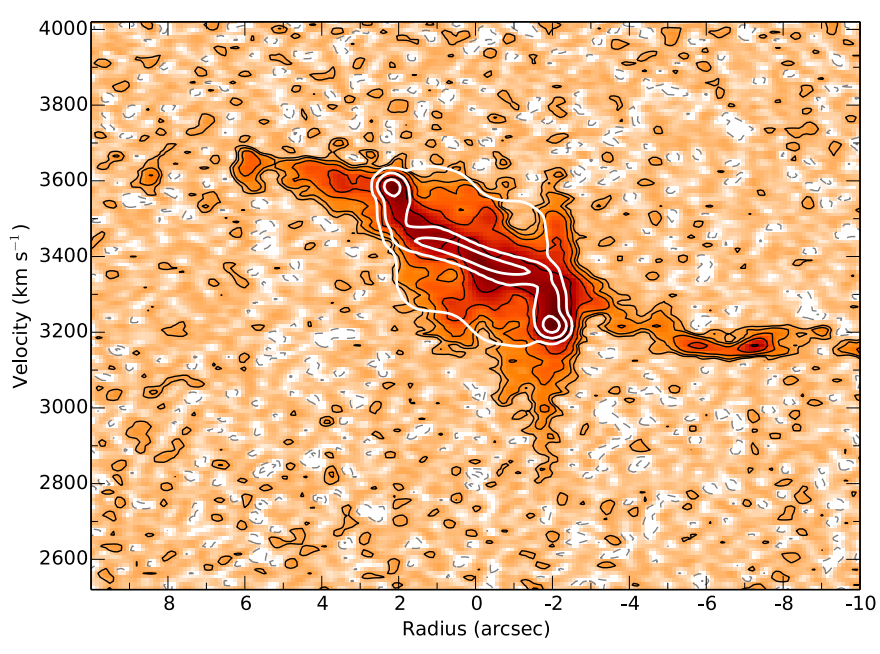

Fig. 8. Position-velocity diagram, as in Fig. 3, with white contours showing the model described in Sect. 5.2.

We find a good fit to the data using a model as described above for the case that the lateral expansion reaches velocities up to $200 \mathrm{~km} \mathrm{~s}^{-1}$ and that the rotation velocity of $\operatorname{ring} R$ (and of component $O$ ) is $\sim 50 \mathrm{~km} \mathrm{~s}^{-1}$ at $r=2^{\prime \prime}$ (compared to a nominal rotation velocity of $170 \mathrm{~km} \mathrm{~s}^{-1}$ at that radius). Since we assume the jet moves in the plane of the sky, projection effects mean that the $200 \mathrm{~km} \mathrm{~s}^{-1}$ for the lateral outflow is a lower limit. We find it necessary, in order to reproduce the large line widths observed for component $O$, to include that this component exhibits a range of expansion velocities, from $0 \mathrm{~km} \mathrm{~s}^{-1}$ to $200 \mathrm{~km} \mathrm{~s}^{-1}$. We chose to implement this by letting the lateral expansion velocity depend linearly on the lateral distance from the jet, but other choices, such as, e.g. a turbulent medium with a large spread in velocity uncorrelated with position, gives very similar results.

Figure 8 shows the observed $p v$ diagram with contours superposed on it based on the above model. Given that we only seek qualitative agreement with the data, we only draw those contours that indicate the qualitative properties of the model. Because we assume the spatial brightness distribution to be uniform for $O$ and $R$, the brightness distribution in the $p v$ diagram is the result of kinematics and geometry, rather than structure in the density or emissivity of the gas disk. Our model reproduces the $\mathrm{S}$-shape of the bright inner feature very well. Although the physical brightness of the affected regions $O$ and $R$ is uniform in the model, the fact that $R$ forms a ring-like structure causes it to project in the $p v$ diagram as a bright ridge. The bright regions just outside the hot spots in our model reproduce $H$. Although component $O$ has, in the plane of the galaxy, the same brightness as $R$, it appears with lower brightness in Fig. 8 because the same amount of emission is spread over a much larger velocity range. The match for component $O$ is also very acceptable, except for the extreme velocities near the western hot spot $(F O)$. Within the framework of our type of model, this can be alleviated by choosing higher lateral expansion velocities at these locations. This is justified because at this location the most intense jet-ISM interaction is occurring, as evidenced by the very bright radio continuum emission from this location. We suggest, similar to Morganti et al. (1998, 2007), Oosterloo et al. (2000), Tadhunter et al. (2014), that at the $\mathrm{W}$ hot spot, the jet is directly interacting with a cloud from the ISM.

We have considered several variations of the model given above. For example, one can assume that the expansion of component $O$ is radial from the centre instead of laterally from the jet. This would apply for a case where the outflow is driven by the central AGN. We find, however, that these models do not reproduce the observed $p v$ diagram very well. In particular, in this kind of model, one would expect the observed anomalous velocities of component $O$ to be largest near the centre and be close to zero near the hot spots. This is quite different from what we observe. Another aspect we varied is the shape of the region containing component $O$ and of the ring $R$, i.e. whether it is circular or elliptical. Although this also depends on the radial density distribution, we found that it is much easier to explain there is no obvious observed radial trend in the brightness of $R$ and $O$ with models where $R$ and $O$ have an elliptical, rather than circular, shape. This can be roughly understood by considering the extreme case of $R$ being a rectangle in which case obviously there is no radial dependence introduced by geometry.

\subsection{Hitting and pushing: the two effects of the radio jet}

The observed distribution and kinematics of the cold molecular gas, as well as the model presented above, support the idea that the radio plasma jet is driving the bulk of the gas outflow.

In this respect, the model follows the idea at the base of the simulations of Wagner \& Bicknell (2011) and Wagner et al. (2012). As mentioned above, these simulations describe the effects of a newly formed radio jet when moving though a dense clumpy medium. Although the clouds initially have densities and temperatures that correspond to a molecular phase, the simulations do not include the complete cooling of the gas and, therefore, they do not follow exactly the same phase of the gas that we observe. However, although a direct comparison of the physical conditions of the gas is not possible, the kinematics of the gas in the observations and model presented here appears to compare remarkably well with the predictions from the numerical simulations.

In our case, the clumpy medium is indeed traced by the bright $\mathrm{CO}$ around the radio jet. As illustrated by, e.g. Fig. 4 in Wagner \& Bicknell (2011), the expanding, quasi-spherical energy bubble is responsible for pushing the clouds outwards. Gas outflowing under the effect of the expanding cocoon is expected to remain visible all along the jet, in the same way as we observed in IC 5063.

Interestingly, this causes the jet-ISM interaction to have an impact over a much broader region than the direct cone of the jet. This is an important element, which makes this kind of interaction particularly relevant in the context of feedback effects.

The kind of strong impact of a jet we observe would be expected when a newly formed radio jet expands into the rich ISM surrounding a young radio source. This has indeed been observed in ionized gas and $\mathrm{HI}$ in young radio sources (Holt et al. 2009; Shih et al. 2013; Gereb et al. 2015). Although we do not know whether IC 5063 is indeed a young radio source, the particular conditions in IC 5063 in which the jet is expanding in the plane of the large-scale gas disk of the host galaxy, likely makes this object an ideal target to test this scenario.

The velocities of the molecular gas observed with ALMA match those obtained by the simulations for jets of comparable power to that in IC 5063. In addition to this, the velocity field expected in these models, as shown in Fig. 4 of Wagner \& Bicknell (2011), shows that higher velocities are expected at the location where the jet is interacting directly with the ISM, as occurs in the $\mathrm{W}$ lobe. We claim that at this location we are seeing the direct interaction of the jet with a large molecular cloud, which is suggested to be present by the NICMOS 
results from Kulkarni et al. (1998). The distribution of clouds in the surrounding ISM is likely not symmetrical near the two lobes, which could explain why the situation in the E lobe is less spectacular.

\subsection{The power source of the outflow}

In the preceding sections, we have shown that a plasma jet expanding and interacting in a gas-rich, clumpy medium appears to be a good explanation for the morphology and kinematics of the gas as observed in our ALMA data. This implies that the radio plasma jet is the main driver of the gas outflow. However, IC 5063 also has a strong (optical) AGN and, therefore, we now discuss the phenomena from an energy point of view.

Using the masses of the outflowing gas given in Sect. 4.4, we derive the mass outflow rate using $\dot{M}=M / \tau_{\text {dyn }}$, where $M$ is the mass of the outflowing gas and $\tau_{\text {dyn }}$ a timescale for the outflow. Given the complex distribution of the outflowing gas, a number of assumptions need to be made. In particular, $\tau_{\text {dyn }}$ was derived using an intermediate distance from the core of $0.3 \mathrm{kpc}$ and an average velocity of the outflowing gas of $200 \mathrm{~km} \mathrm{~s}^{-1}$, which is more typical of the gas along the jet than the extreme values at the location of the $\mathrm{W}$ lobe. This yields $\tau_{\text {dyn }} \simeq 1.5 \times 10^{6} \mathrm{yr}$, and we obtained $\dot{M}$ to lie in the range 12 to $30 M_{\odot} \mathrm{yr}^{-1}$, depending on the assumed $\mathrm{CO}$-to- $\mathrm{H}_{2}$ conversion factor.

The kinetic power of the outflowing gas can be estimated using the formula in Holt et al. (2006) that includes, unlike the calculations in Cicone et al. (2014), both the radial and turbulent components in the gas motion. Following Holt et al. (2006), we assumed that the relatively large line width of the outflowing gas reflects a turbulent motion that is present at all locations in the outflow region. Thus, the component of turbulence is represented by the full width at half maximum (FWHM) of the lines,

$\dot{E}=6.34 \times 10^{35} \frac{\dot{M}}{2}\left(v_{\text {out }}^{2}+\frac{v_{\text {turb }}^{2}}{5.55}\right) \quad \mathrm{erg} \mathrm{s}^{-1}$,

where we have assumed $v_{\text {out }}=200 \mathrm{~km} \mathrm{~s}^{-1}$ and $v_{\text {turb }} \sim F W H M \sim$ $100 \mathrm{~km} \mathrm{~s}^{-1}$. These values are giving us a very conservative estimate of the kinetic power of the outflow. Combining the H I and $\mathrm{CO}$ components, this gives a kinetic power of the outflow of $\sim 5 \times 10^{42} \mathrm{erg} \mathrm{s}^{-1}$.

We can now compare this with the bolometric luminosity of the AGN and the jet power. The bolometric luminosity of the AGN is estimated to be $L_{\mathrm{AGN}}^{\mathrm{bol}}=7.7 \times 10^{44} \mathrm{erg} \mathrm{s}^{-1}$ (Morganti et al. 2007), thus the optical AGN is in principle capable of driving the outflow if the efficiency is higher than $0.6 \%$.

We estimate the jet power using the formulae given in Willot et al. (1999), Wu (2009), and Cavagnolo et al. (2010), and we obtain values between $5 \times 10^{43} \mathrm{erg} \mathrm{s}^{-1}$ and of $9 \times 10^{43} \mathrm{erg} \mathrm{s}^{-1}$ (using a conservative value of $f=10$ for the normalisation uncertainty factor introduced by Willot et al. 1999; see also Wu 2009 for more details). This indicates that the coupling between the energy transmitting to the medium by the jet and the ISM should have high efficiency (between 0.05 and 0.1 ) to drive the observed outflow.

Both phenomena, AGN and radio jet, can power the outflow. Despite this, the observed kinematics suggests that the radio jet plays a prominent role in driving the outflow. This may indicate that either it has a higher efficiency/coupling, or that perhaps both are at work in the inner regions while the radio jet dominates at larger distances.

It is worth noting that IC 5063 was part of the sample included in the analysis of Cicone et al. (2014). IC 5063, however, seems to deviate from the correlation they found between the molecular outflow rate and the AGN luminosity (their Fig. 9). This correlation was taken as a signature of the optical AGN driving the outflow in the sample of local ULIRGs and QSO hosts considered by these authors. The fact that IC 5063 does not lie on this correlation indicates that the radio jet indeed could play a role in IC 5063.

\subsection{Interaction, shocks, and cold gas}

Although the model described above can explain the kinematics of the gas quite well, questions remain about how cold gas can be associated with these fast and massive outflows, since shocks accelerate the gas and may heat it to $10^{6}-10^{7} \mathrm{~K}$. Molecules should dissociate at these temperatures (Hollenbach \& McKee 1989). In the case of shocks produced by winds from the accretion disks, Zubovas \& King (2014) proposed that radiative cooling is fast enough to reform molecules in a large fraction of the outflowing material on a short timescale. They assume that, because of Rayleigh-Taylor instabilities, the cool gas should collapse into clumps, which are then entrained in the hot-diffuse medium. Key for the success of this type of model is the timescale over which the clumps can form.

A similar scenario, albeit with shocks driven by the radio plasma, has been proposed for IC 5063 to explain the warm molecular gas kinematics (Tadhunter et al. 2014) and for the case of 4C 12.50 to explain the outflowing H I (Morganti et al. 2013a). In the latter study, a cooling time of the order of $10^{4}$ years was derived for the outflowing cloud. This timescale is comparable to the age of the radio source, suggesting that the gas indeed has time to cool during the process of being expelled. Finally, Guillard et al. (2009, 2012) proposed that the turbulent gas becomes thermally unstable and cools as it moves. Therefore the molecular gas that results from the post-shock cooling has the momentum of the tenuous gas that has been accelerated to high velocities. This may explain why the molecular gas is characterised by these high velocities.

To confirm these scenarios, the emissivity of gas strongly disturbed by the passage of a radio jet and associated shocks needs to be followed with simulations. This has been investigated in a number of studies, although with particular emphasis on young stellar and Herbig-Haro objects (e.g. Chernin et al. 1994; de Gouveia Dal Pino 1999; Raga et al. 2002). Investigations, using a similar approach, to trace the cooler gas by expanding to extragalactic sources the simulations of relativistic jets presented in Rocha da Silva (2015), are now in progress. Preliminary results (Falceta-Gonçalves \& de Gouveia Dal Pino, priv. comm.) show that a relativistic jet entering a denser environment is developing a turbulent cocoon. The consequent fast cooling, associated with the turbulence (plus entrainment), results in a mix of gas phases (from hot to cold) as is observed. Most importantly, the simulations show, to first order, that the cooler gas is also characterised by high velocities. Thus, despite the simplicity of the assumptions made (i.e. homogeneous ambient medium), the shocked ambient material deposited into the cocoon around the jet includes a cool component (in addition to the warm and hot) with large turbulent velocities, in agreement with the observations.

The results of our observations can also be used to investigate alternative scenarios: for example, slow entrainment by the jet that would not dissociate the molecules. However, if the warm $\mathrm{H}_{2}$ is also heated during this entrainment, the mass discrepancy between the warm $\mathrm{H}_{2}\left(M_{\mathrm{H}_{2}} \sim 8.2 \times 10^{2} M_{\odot}\right.$, Tadhunter et al. 2014), and cooler components derived from the $\mathrm{CO}(2-1)$ 
observations presented here, is difficult to explain. Alternatively, if the heating of the clouds (and of the warm molecular gas) is done by the AGN radiation field (i.e. in a similar way as seen in radiatively excited molecular clouds, where the warm $\mathrm{H}_{2}$ forms a skin around the colder molecular material deeper in the clouds). Our new $\mathrm{CO}(2-1)$ observations make this possibility unlikely, however, because it is difficult to explain how more than $10^{7} M_{\odot}$ of molecular gas (equivalent to an extremely massive giant molecular cloud) can be accelerated to the observed high velocities without heating the molecules and destroying the clouds. Thus, we conclude that the most likely explanation for the outflowing molecular gas in IC 5063 is rapid post-shock cooling.

\section{Conclusions}

The ALMA data of IC 5063 presented here provide a unique, high-resolution $3 \mathrm{D}$ view of the molecular gas in one of the best examples of an on-going interaction between the radio jet and the ISM. Because of its proximity, and its geometry, this object is ideal for studying the impact of radio jets on the surrounding medium. A wealth of data, including the warm molecular gas, H I and ionized gas, are already available for this object. However, the ALMA data provide a much more complete and complex picture and allow us to trace this interaction in much more detail (down to scales of 0.5 arcsec, i.e. $100 \mathrm{pc}$ ) compared to what is available for the other phases of the gas. The results presented here further confirm the multi-phase nature of AGNdriven outflows and the significant mass of the cold gaseous component associated with it.

From these data, we found kinematically disturbed gas distributed all along the radio emission, with velocities strongly deviating from the regularly rotating gas. This illustrates that gas outflows are produced not only where the jet directly interacts with the ISM, but that the effects occur over a much larger volume therefore making its impact larger, as already shown by numerical simulations (e.g. Wagner \& Bicknell 2011; Wagner et al. 2012).

The region showing the disturbed kinematics of the gas is cospatial with the radio plasma and it also appears much brighter than the gas of the larger-scale, regularly rotating component of molecular gas. This inner, brighter region of $\mathrm{CO}(2-1)$ is elongated along the radio axis and wraps around the radio continuum emission. The brightest $\mathrm{CO}(2-1)$ emission is co-spatial with the bright, western radio lobe and has the largest velocities deviating from regular rotation. A simple model assuming lateral expansion of the gas caused by the cocoon around the radio jet, describes the observed kinematics remarkably well.

Taking all this together, we argue that the distribution and kinematics of the molecular gas support the idea of the jet interacting with a clumpy medium and pushing it outwards. In addition to the outflowing gas, this results in the creation of a ring of gas, which represents the interface between the region affected by the radio plasma and the undisturbed disk at larger radius. At least at the location of the western hot spot, the jet is directly interacting with a gas cloud, causing the very large outflow velocities observed. This picture is fully consistent with the numerical simulations of Wagner \& Bicknell (2011) and Wagner et al. (2012) of which all main components are clearly identifiable in the data.

Even assuming the most conservative value of the conversion CO-to- $\mathrm{H}_{2}$, we find that the mass of the outflowing gas is between 1.8 and $4.5 \times 10^{7} M_{\odot}$, thus much larger than the outflow observed in any other gas phase. The mass outflow rates we derive are in the range $12-30 M_{\odot} \mathrm{yr}^{-1}$.

Looking at the energetics, it appears that both the bolometric luminosity of the $\mathrm{AGN}$, as well as the jet power, are large enough to drive the outflow. The finding, based on the kinematics, that the radio plasma plays a dominant role (at least at radii larger than $100 \mathrm{pc}$ ), could suggest a higher efficiency for this mechanism.

Particularly relevant from this study is the conclusion that the effect of the radio plasma can also be significant in objects such as IC 5063, which are often considered radio quiet. A number of cases in which this situation may occur have recently been identified; e.g. NGC 1266 (Alatalo et al. 2011; Nyland et al. 2013); NGC 1433 (Combes et al. 2013); J1430+1339, nicknamed the "Teacup AG" (Harrison et al. 2015). However, direct evidence that the radio plasma is at the origin of these outflows of cold gas is, so far, not available in all these cases. Another case is M 51 in which signatures of the interaction between a weak radio jet and the ISM have been found in the molecular gas. A high-density region traced by HCN has been detected along the weak radio jet of this nearby object. This region shows a gradient in the $\mathrm{HCN} / \mathrm{CO}$ ratio, with decreasing values for larger distances from the jet (Matsushita et al. 2015). This points to the effect of the jet pushing and compressing the surrounding medium. If the possibility of weak radio jets driving massive gas outflows because of their high coupling efficiency is confirmed for more objects, it would make even "wimpy" radio jets relevant for the fate of gas in galaxies. The fact that these weak radio jets are more common than the powerful jets can have implications for the role of feedback in galaxy evolution.

Another interesting point in the context of feedback effects is the fate of the kinematically disturbed gas. The observed outflow velocities of most of the disturbed gas are only $\sim 200 \mathrm{~km} \mathrm{~s}^{-1}$. Under these conditions, the gas does not leave the galaxy, but is most likely just relocated in a process comparable to a "galactic fountain". Of the gas with disturbed kinematics, at most only a very small fraction may have velocities high enough to leave the galaxy, which is a similar situation as observed in, e.g. NGC 1266 (Alatalo et al. 2015). However, the injection of large amounts of energy in the gas disk may have a similar effect as increasing the turbulence of the gas, a process that is also thought to inhibit star formation (see the case of 3C 326, Guillard et al. 2015).

In summary, the ALMA data presented here for IC 5063 show how spatially resolved observations are key to being able to determine which mechanism, radiation, or jet, drives the observed kinetic feedback. Hopefully, expanding this kind of work to include more objects will finally allow us to quantify the impact of an AGN on the ISM of the host galaxy.

Acknowledgements. We thank Alex Wagner, Elisabete de Gouveia Dal Pino and Diego Falceta-Goncalves for their input and useful discussions. This paper makes use of the following ALMA data: ADS/JAO.ALMA\#2012.1.00435.S. ALMA is a partnership of ESO (representing its member states), NSF (USA), and NINS (Japan), together with NRC (Canada) and NSC, and ASIAA (Taiwan), in cooperation with the Republic of Chile. The Joint ALMA Observatory is operated by ESO, AUI/NRAO and NAOJ. R.M. gratefully acknowledges support from the European Research Council under the European Union's Seventh Framework Programme (FP/2007-2013)/ERC Advanced Grant RADIOLIFE-320745.

\section{References}

Alatalo, K., Blitz, L., Young, L. M., et al. 2011, ApJ, 735, 88

Alatalo, K., Davis, T. A., Bureau, M., et al. 2013, MNRAS, 432, 1796 
R. Morganti et al.: The fast molecular outflow in the Seyfert galaxy IC 5063 as seen by ALMA

Alatalo, K. Lacy, M., Lanz, L., et al. 2015, ApJ, 798, 31

Bayet, E., Williams, D. A., Hartquist, T. W., \& Viti, S. 2011, MNRAS, 414, 1583

Best, P. N., Kauffmann, G., Heckman, T. M., et al. 2005, MNRAS, 362, 25

Binney, J., \& Tremaine, S. 2008, Galactic Dynamics, second edition (Princeton: Princeton University Press)

Bîrzan, L., McNamara, B. R., Nulsen, P. E. J., Carilli, C. L., \& Wise, M. W. 2008, ApJ, 686, 859

Bolatto, A. D., Wolfire, M., \& Leroy, A. K. 2013, ARA\&A, 51, 207

Cappellari, M., Scott, N., Alatalo, K., et al. 2013, MNRAS, 432, 1709

Cavagnolo, McNamara, B. R., Nulsen, P. E. J., et al. 2010, ApJ, 720, 1066

Chernin, L., Masson, C., Gouveia dal Pino, E. M., \& Benz, W. 1994, ApJ, 426, 204

Cicone, C., Maiolino, R., Sturm, E., et al. 2014, A\&A, 562, A21

Combes, F. 2015, Proc. IAU Symp., 309, 182

Combes, F., García-Burillo, S., Casasola, V., et al. 2013, A\&A, 558, A124

Colina, L., Sparks, W. B., \& Macchetto, F. 1991, ApJ, 370, 102

Costa, T., Sijacki, D., \& Haehnelt, M. G. 2014, MNRAS, 444, 2355

Dasyra, K. M., \& Combes, F. 2011, A\&A, 533, L10

Dasyra, K. M., \& Combes, F. 2012, A\&A, 541, L7

Davis, T. A., Alatalo, K., Bureau, M., et al. 2013, MNRAS, 429, 534

de Gouveia Dal Pino, E. M. 1999, ApJ, 526, 862

Fabian, A. 2013, ARA\&A, 50, 455

Ferland, G. J., Fabian, A. C., Hatch, N. A., et al. 2009, MNRAS, 392, 1475

Feruglio, C., Maiolino, R., Piconcelli, E., et al. 2010, A\&A, 518, L155

García-Burillo, S., Combes, F., Usero, A., et al. 2014, A\&A, 567, A125

Geach, J. E., Hickox, R. C., Diamond-Stanic, A. M., et al. 2014, Nature, 516, 68

Geréb, K., Morganti, R., \& Oosterloo, T. A. 2014, A\&A, 569, A35

Geréb, K., Maccagni, F., Morganti, R., \& Oosterloo, T. 2015, A\&A, 575, A44

Guillard, P., Boulanger F., Pineau Des Forêts, G., \& Appleton, P. N. 2009, A\&A, 502,515

Guillard, P., Ogle, P. M., Emonts, B. H. C, et al., 2012, ApJ, 747, 95

Guillard, P., Boulanger, F., Lehnert, M. D., et al. 2015, A\&A, 574, A32

Harrison, C. M., Thomson, A. P., Alexander, D. M., et al. 2015, ApJ, 800, 45

Hollenbach, D., \& McKee, C. F. 1989, ApJ, 342, 306

Holt, J., Tadhunter, C., Morganti, R., et al. 2006, MNRAS, 370, 1633

Holt, J., Tadhunter, C. N., \& Morganti, R. 2009, MNRAS, 400, 589

Józsa, G. I. G., Kenn, F., Klein, U., \& Oosterloo, T. A. 2007, A\&A, 468, 731

Kanekar, N., \& Chengalur, J. N. 2008, MNRAS, 384, 6

Krause, M., Fendt, C., \& Neininger, N. 2007, A\&A, 467, 1037

Kulkarni, V. P., Calzetti, D., Bergeron, L., et al. 1998, ApJ, 492, L121

Loenen, A. F., Spaans, M., Baan, W. A., \& Meijerink, R. 2008, A\&A, 488, L5

Mahony, E., Morganti, R., Emonts, B., Oosterloo, T., \& Tadhunter, C. 2013, MNRAS, 435, L58

Maloney, Ph. R., Hollenbach, D. J., \& Tielens, A. G. G. M. 1996, ApJ, 466, 561
Martin, P., Roy, J.-R., Noreau, L., \& Lo, K.Y. 1989, ApJ, 345, 707

Matsushita, S., Muller, S., \& Lim, J. 2007, A\&A, 468, L49

Matsushita, S., Trung, D.-V.-, Boone, F., et al. 2015, ApJ, 799, 26

McMullin, J. P., Waters, B., Schiebel, D., Young W., \& Golap, K. 2007, ASP Conf. Ser., 376, 127

McNamara, B. R., \& Nulsen, P. E. J. 2012, New J. Phys., 14, 055023

Meijerink, R., Spaans, M., Israel, F. P., et al. 2007, A\&A, 461, 793

Meijerink, R., Spaans, M., Loenen, A. F., \& van der Werf, P. P. 2011, A\&A, 525, A119

Morganti, R. 2015, Proc. IAU Symp., 313, 283

Morganti, R., Oosterloo, T. A., \& Tsvetanov, Z. 1998, AJ, 115, 915

Morganti, R., Tadhunter, C. N., \& Oosterloo, T. A. 2005a, A\&A, 444, L9

Morganti, R., Oosterloo, T. A., Tadhunter, C. N., van Moorsel, G., \& Emonts, B. 2005b, A\&A, 439, 521

Morganti, R., Holt, J., Saripalli, L., Oosterloo, T. A., \& Tadhunter, C. N. 2007, A\&A, 476, 735

Morganti, R., Fogasy, J., Paragi, Z., Oosterloo, T., Orienti, M. 2013a, Science, 341,1082

Morganti, R., Frieswijk, W., Oonk R., Oosterloo, T., \& Tadhunter, C. 2013b, A\&A, 552, L4

Nyland, K., Alatalo, Katherine, Wrobel, J. M., et al. 2013, ApJ, 779, 173

Oosterloo, T., Morganti, R., Tzioumis, A., et al. 2000, AJ, 119, 2085

Raga, A. C., de Gouveia Dal Pino, E. M., Noriega-Crespo, A., Mininni, P. D., \& Velázquez, P. F. 2002, A\&A, 392, 267

Rocha da Silva, G., Falceta-Gonçalves, D., Kowal, G., \& de Gouveia Dal Pino, E. M. 2015, MNRAS, 446, 104

Sault, R. J., Teuben, P. J., \& Wright, M. C. H., 1995, ASP Conf. Ser., 77, 433

Serra, P., Oosterloo, T., Morganti, R., et al. 2012, MNRAS, 422, 1835

Serra, P., Oser, L., Krajnović, Davor, et al. 2014, MNRAS, 444, 3388

Shih, H.-Y., Stockton, A., \& Kewley, L. 2013, ApJ, 772, 138

Sternberg, A., Dalgarno, A., Lepp, S., et al. 1987, ApJ, 320, 676

Tadhunter, C. 2008, Mem. Soc. Astron. It., 79, 1205

Tadhunter, C., Morganti, R., Rose, M., Oonk, J. B. R., \& Oosterloo, T. 2014, Nature, 511, 440

Tielens, A. G. G. M., \& Hollenbach, D. 1985, ApJ, 291, 722

van Driel, W., \& van Woerden, H. 1991, A\&A, 243, 71

Wagner, A. Y., \& Bicknell, G. V. 2011, ApJ, 728, 29

Wagner, A. Y., Bicknell, G. V., \& Umemura, M. 2012, ApJ, 757, 136

Wiklind, T., Combes, F., \& Henkel, C. 1995, A\&A, 297, 643

Willott, C., Rawlings, S., Blundell, K., \& Lacy, M. 1999, MNRAS, 309, 1017

Wu, Q. 2009, MNRAS, 398, 1905

Zubovas, K., \& King, A. 2012, ApJ, 745, L34

Zubovas, K., \& King, A. 2014, MNRAS, 439, 400

Young, L. M., Bureau, M., Davis, T. A., et al. 2011, MNRAS, 414, 940 